\title{
Enumeration of cat1-groups of low order
}

Wensley, Christopher D.; Alp, Murat

\section{International Journal of Algebra and Computation}

\author{
DOI: \\ $10.1142 / \mathrm{S} 0218196700000170$
}

Published: 01/01/2000

Peer reviewed version

Cyswllt i'r cyhoeddiad / Link to publication

Dyfyniad o'r fersiwn a gyhoeddwyd / Citation for published version (APA):

Wensley, C. D., \& Alp, M. (2000). Enumeration of cat1-groups of low order. International Journal of Algebra and Computation, 10(04), 407-424. https://doi.org/10.1142/S0218196700000170

\footnotetext{
Hawliau Cyffredinol / General rights

Copyright and moral rights for the publications made accessible in the public portal are retained by the authors and/or other copyright owners and it is a condition of accessing publications that users recognise and abide by the legal requirements associated with these rights.

- Users may download and print one copy of any publication from the public portal for the purpose of private study or research.

- You may not further distribute the material or use it for any profit-making activity or commercial gain

- You may freely distribute the URL identifying the publication in the public portal ?
}

Take down policy

If you believe that this document breaches copyright please contact us providing details, and we will remove access to the work immediately and investigate your claim. 


\title{
Enumeration of cat $^{1}$-groups of low order
}

\author{
Murat Alp \\ Dumlupınar Üniversitesi, Fen-Edebiyat Fakültesi \\ Matematik Bölümü, Merkez Kampüs \\ Kütahya, Turkey \\ and \\ Christopher D. Wensley \\ School of Mathematics, University of Wales, \\ Bangor, Gwynedd, LL57 1UT, U.K. \\ (email: c.d.wensley@bangor.ac.uk)
}

January 7, 1999

1991 Mathematics Subject Classification: 13D99, 18-04, 18D35, 20-04.

Keywords: crossed module, cat1-group, derivation, actor.

\begin{abstract}
In this paper we describe a share package XMOD of functions for computing with finite, permutation crossed modules, cat $^{1}$-groups and their morphisms, written using the GAP group theory programming language. The category XMod of crossed modules is equivalent to the category Cat1 of cat ${ }^{1}$-groups and we include functions emulating the functors between these categories. The monoid of derivations of a crossed module $\mathcal{X}$, and the corresponding monoid of sections of a cat ${ }^{1}$-group $\mathcal{C}$, are constructed using the Whitehead multiplication. The Whitehead group of invertible derivations, together with the group of automorphisms of $X$, are used to construct the actor crossed module of $X$ which is the automorphism object in XMod. We include a table of the 350 isomorphism classes of cat $^{1}$-structures on groups of order at most 30 .
\end{abstract}




\section{Introduction}

Our aim in this paper is to describe a share package XMOD [2] for the GAP [20] computational group theory language. This package implements calculations with finite, permutation crossed modules and their morphisms, and also with the equivalent cat ${ }^{1}$-groups and their morphisms. We also present the results of the computation of all isomorphism classes of cat ${ }^{1}$-group structures on groups of order at most 30 .

This is the first computational contribution to the general programme of "higher-dimensional group theory" described, for example, by Brown in [4] and [5]. The 2-dimensional part of this programme is concerned with group objects in the category of groupoids, and these objects may equivalently be considered as crossed modules or cat $^{1}$-groups.

Whereas the theory of groups started with groups of permutations and developed into abstract groups and topological groups, the impetus for the study of "2-dimensional groups" comes from algebraic topology, in describing the homotopy double groupoid of a based pair of spaces. Nevertheless, the algebraic motivation is equally compelling: the automorphism group $\operatorname{Aut}(G)$ of a group $G$ is most clearly exhibited as part of the automorphism crossed module (inn : $G \rightarrow \operatorname{Aut}(G)$ ) determined by the inner automorphism map.

The term crossed module was introduced by J. H. C. Whitehead in [21]. See [6, 7, 8] and [12] for a variety of applications. In [16] Loday reformulated the notion of a crossed module as a cat ${ }^{1}$ group (though he used the term 1-cat group) and showed that the category XMod is equivalent to the category Cat1. Loday also generalised the notion of cat $^{1}$-group to that of cat $^{n}$-group, for all $n \geq 1$. Crossed modules and their higher analogues were considered by Ellis in [13]. A proof that the categories of cat $^{n}$-groups and crossed $n$-cubes are equivalent was given by Ellis and Steiner in [14].

The category XMod is also equivalent to the category GpGpd of group-groupoids and to the category of 1-truncated simplicial groups with trivial Moore complex (see [3, 16]). These structures are not included in the current version of the package.

In section 2 we recall the basic properties of crossed modules and their derivations and of cat ${ }^{1}$ groups and their sections. In section 3 we describe the implementation of these structures in GAP and include a short example illustrating how the package is used. In section 4 we discuss some of the algorithms used to compute lists of derivations, sections and cat ${ }^{1}$-structures.

In section 5 we tabulate, for groups $G$ of order at most 30 , the order of $\operatorname{End}(G)$; the number of idempotent endomorphisms; the number of cat $^{1}$-structures on $G$; and the number of isomorphism classes of these structures.

The authors wish to thank R. Brown and T. Porter for many profitable discussions concerning the algebraic constructions. Considerable help with the implementation has been given by many members of the GAP team at Aachen, led by J. Neubüser, and by D. Holt who is the appointed editor for the package. The first author is supported by Dumlupinar University, Turkey, for studies at Bangor culminating in his thesis [1]. The second author is grateful for the invitation to attend the GAP4 workshop in September 1996 and for the hospitality provided. The computational work was done using a Digital Alpha computing laboratory set up with a SERC grant (GR/J63552) in 1993. 


\section{Crossed Modules and Cat ${ }^{1}$-Groups}

In this section we recall the descriptions of three equivalent categories: XMod, the category of crossed modules and their morphisms; Cat1, the category of cat ${ }^{1}$-groups and their morphisms; and GpGpd, the subcategory of groups in the category Gpd of groupoids. We also describe functors between these categories which exhibit the equivalences. We state the axioms using right actions, since this is the convention used by most computational group packages, but all functions will be written on the left.

A crossed module $\mathcal{X}=(\partial: S \rightarrow R)$ consists of a group homomorphism $\partial$, called the boundary of $\mathcal{X}$, together with an action $\alpha: R \rightarrow \operatorname{Aut}(S)$ satisfying, for all $s, s^{\prime} \in S$ and $r \in R$,

$$
\begin{aligned}
\text { XMod 1: } & \partial\left(s^{r}\right) & =r^{-1}(\partial s) r \\
\text { XMod 2: } & s^{\partial s^{\prime}}= & s^{\prime-1} s s^{\prime} .
\end{aligned}
$$

The kernel of $\partial$ is abelian and the image of $\partial$ is normal in $R$.

Standard constructions for crossed modules include the following:

1. A conjugation crossed module is an inclusion of a normal subgroup $S \unlhd R$, where $R$ acts on $S$ by conjugation.

2. An automorphism crossed module has as range a subgroup $R$ of the automorphism group Aut $(S)$ of $S$ which contains the inner automorphism group $\operatorname{Inn}(S)$ of $S$. The boundary maps $s \in S$ to the inner automorphism of $S$ by $s$.

3. An $R$-Module crossed module has an $R$-module as source and $\partial=0$.

4. Any homomorphism $\partial: S \rightarrow R$, with $S$ abelian and im $\partial$ in the centre of $R$, provides a crossed module with $R$ acting trivially on $S$.

5. A central extension crossed module has as boundary a surjection $\partial: S \rightarrow R$ with central kernel, where $r \in R$ acts on $S$ by conjugation with $\partial^{-1} r$.

6. The direct product of $\mathcal{X}_{1}=\left(\partial_{1}: S_{1} \rightarrow R_{1}\right)$ and $\mathcal{X}_{2}=\left(\partial_{2}: S_{2} \rightarrow R_{2}\right)$ is $\mathcal{X}_{1} \times \mathcal{X}_{2}=\left(\partial_{1} \times \partial_{2}:\right.$ $\left.S_{1} \times S_{2} \rightarrow R_{1} \times R_{2}\right)$ with $R_{1}, R_{2}$ acting trivially on $S_{2}, S_{1}$ respectively.

A morphism between two crossed modules $\mathcal{X}_{1}$ and $\mathcal{X}_{2}$ is a pair $(\sigma, \rho)$, where $\sigma: S_{1} \rightarrow S_{2}$ and $\rho: R_{1} \rightarrow R_{2}$ are homomorphisms satisfying

$$
\partial_{2} \sigma=\rho \partial_{1}, \quad \sigma\left(s^{r}\right)=(\sigma s)^{\rho r} .
$$

When $\mathcal{X}_{2}=\mathcal{X}_{1}$ and $\sigma, \rho$ are automorphisms then $(\sigma, \rho)$ is an automorphism of $\mathcal{X}_{1}$. The group of automorphisms is denoted by $\operatorname{Aut}\left(\mathcal{X}_{1}\right)$.

The Whitehead monoid $\operatorname{Der}(\mathcal{X})$ of $\mathcal{X}$ was defined in [22] to be the monoid of all derivations from $R$ to $S$, that is the set of all maps $R \rightarrow S$, with composition $\circ$, satisfying

$$
\begin{array}{rrrl}
\text { Der 1: } & \chi(q r) & =(\chi q)^{r}\left(\chi_{r}\right) \\
\text { Der 2: } & \left(\chi_{1} \circ \chi_{2}\right)(r) & =\left(\chi_{1} r\right)\left(\chi_{2} r\right)\left(\chi_{1} \partial \chi_{2} r\right) .
\end{array}
$$


Invertible elements in the monoid are called regular. The Whitehead group $\mathcal{W}(\mathcal{X})$ is the group of $\operatorname{Der}(\mathcal{X})$. The actor of $\mathcal{X}$ is a crossed module $(\Delta: \mathcal{W}(\mathcal{X}) \rightarrow \operatorname{Aut}(\mathcal{X}))$ which was shown by Lue and Norrie, in $[17,18,19]$, to be the automorphism object of $\mathcal{X}$ in the category XMod. Gilbert, in [15], has discussed a connection between derivations and group extensions.

In [16] Loday reformulated the notion of a crossed module as a cat ${ }^{1}$-group, namely a group $G$ with a pair of endomorphisms $t, h: G \rightarrow G$ having a common image $R$ and satisfying certain axioms. We prefer a definition in which a cat ${ }^{1}$-group $\mathcal{C}=(e ; t, h: G \rightarrow R)$ has source group $G$, range group $R$, and three homomorphisms: two surjections $t, h: G \rightarrow R$ and an embedding $e: R \rightarrow G$ satisfying:

Cat 1: $t e(r)=h e(r)=r$ for all $r \in R$,

Cat 2: $\quad[\operatorname{ker} t, \operatorname{ker} h]=\left\{1_{G}\right\}$.

The maps $t, h$ are usually referred to as the source and target, but we choose to call them the tail and head of $\mathcal{C}$, because source is the GAP term for the domain of a function.

A morphism $\mathcal{C}_{1} \rightarrow \mathcal{C}_{2}$ of cat ${ }^{1}$-groups is a pair $(\gamma, \rho)$ where $\gamma: G_{1} \rightarrow G_{2}$ and $\rho: R_{1} \rightarrow R_{2}$ are homomorphisms satisfying

$$
h_{2} \gamma=\rho h_{1}, \quad t_{2} \gamma=\rho t_{1}, \quad e_{2} \rho=\gamma e_{1} .
$$

An arbitrary cat ${ }^{1}$-group $\mathcal{C}=(e ; t, h: G \rightarrow R)$ is isomorphic to the cat ${ }^{1}$-group $\mathcal{C}^{\prime}=\left(e^{\prime} ; t^{\prime}, h^{\prime}:\right.$ $R \ltimes S \rightarrow R$ ) where $S=\operatorname{ker} t, R$ acts on $S$ by

$$
s^{r}=s^{e r}=(e r)^{-1} s(e r)
$$

and the semidirect product $R \ltimes S$ has composition and inverse given by

$$
\left(r_{1}, s_{1}\right)\left(r_{2}, s_{2}\right)=\left(r_{1} r_{2}, s_{1}^{r_{2}} s_{2}\right), \quad(r, s)^{-1}=\left(r^{-1},\left(s^{-1}\right)^{r^{-1}}\right) .
$$

The homomorphisms in $\mathcal{C}^{\prime}$ are given by

$$
t^{\prime}(r, s)=r, \quad h^{\prime}(r, s)=r(\partial s), \quad e^{\prime} r=(r, 1)
$$

and the isomorphism $\left(\theta, \operatorname{id}_{R}\right): \mathcal{C}^{\prime} \rightarrow \mathcal{C}$ is given by

$$
\theta: R \ltimes S \rightarrow G, \quad(r, s) \mapsto(e r) s
$$

with inverse

$$
\theta^{-1}: G \rightarrow R \ltimes S, \quad g \mapsto\left(t g,\left(e t g^{-1}\right) g\right) .
$$

The crossed module $\mathcal{X}=(\partial: S \rightarrow R)$ associated to $\mathcal{C}$ and $\mathcal{C}^{\prime}$ has $\partial=h_{S}$. The cat ${ }^{1}$-group $\mathcal{C}=\mathcal{C}^{\prime}$ associated to $\mathcal{X}=(\partial: S \rightarrow R)$ has $G=R \ltimes S$, where the action is that in $\mathcal{X}$, and homomorphisms given by (2). We denote by $\epsilon$ the inclusion of $S$ in $G$, so that $\partial=h \epsilon$.

The construction for cat $^{1}$-groups equivalent to the derivation of a crossed module is the section, namely a group monomorphism $\xi: R \rightarrow G$ satisfying:

Sect 1: $t \xi(r)=r$ for all $r \in R$. 
The equation

$$
\xi r=(e r)(\epsilon \chi r)=(r, \chi r)
$$

defines a section $\xi$ of $\mathcal{C}$ in terms of a derivation $\chi$ of $\mathcal{X}$, and conversely. These sections form the monoid $\operatorname{Sect}(\mathcal{C})$ of $\mathcal{C}$, whose composition rule we determine from the rule $\operatorname{Der} 2$ for $\operatorname{Der}(\mathcal{X})$ by evaluating:

$$
\begin{aligned}
\left(\xi_{1} \circ \xi_{2}\right) r & =(e r)\left(\epsilon\left(\chi_{1} \circ \chi_{2}\right) r\right) \\
& =(e r)\left(\epsilon \chi_{1} r\right)\left(\epsilon \chi_{2} r\right)\left(\epsilon \chi_{1} h \epsilon \chi_{2} r\right) \\
& =\left(\xi_{1} r\right)\left(e r^{-1}\right)\left(\xi_{2} r\right)\left(e h\left(\epsilon \chi_{2} r\right)^{-1}\right)\left(\xi_{1} h \epsilon \chi_{2} r\right) \\
& =\left(\xi_{1} r\right)\left(e r^{-1}\right)\left(\xi_{2} r\right)\left(e h\left(\left(\xi_{2} r\right)^{-1}(e r)\right)\right)\left(\xi_{1} h\left(\left(e r^{-1}\right)\left(\xi_{2} r\right)\right)\right) \\
& =\left((e r)\left(\xi_{1} r^{-1}\right)\right)^{-1}\left(\left(\xi_{2} r\right)\left(e h \xi_{2} r^{-1}\right)\right)\left((e r)\left(\xi_{1} r^{-1}\right)\right)\left(\xi_{1} h \xi_{2} r\right) .
\end{aligned}
$$

Since $(e r)\left(\xi_{1} r^{-1}\right)$ and $\left(\xi_{1} h \xi_{2} r\right)\left(e h \xi_{2} r^{-1}\right) \in \operatorname{ker} t$ while $\left(\xi_{2} r\right)\left(e h \xi_{2} r^{-1}\right) \in \operatorname{ker} h$, this reduces to

Sect 2: $\quad\left(\xi_{1} \circ \xi_{2}\right) r=\left(\xi_{2} r\right)\left(e h \xi_{2} r^{-1}\right)\left(\xi_{1} h \xi_{2} r\right)=\left(\xi_{1} h \xi_{2} r\right)\left(e h \xi_{2} r^{-1}\right)\left(\xi_{2} r\right)$.

The embedding $e$ is the identity for this composition, and equation (3) determines a monoid isomorphism $\operatorname{Der}(\mathcal{X}) \cong \operatorname{Sect}(\mathcal{C})$. A section is regular when $h \xi$ is an automorphism and the group of regular sections is isomorphic to the Whitehead group.

Each $\chi$ or $\xi$ determines endomorphisms of $R, S, G, \mathcal{X}$ and $\mathcal{C}$, namely

$$
\begin{array}{rll}
\rho & : R \rightarrow R, \quad r \mapsto r(\partial \chi r)=h \xi r, \\
\sigma & : S \rightarrow S, \quad s \mapsto s(\chi \partial s)=s\left(e \partial s^{-1}\right)(\xi \partial s), \\
\gamma \quad: \quad G \rightarrow G, \quad g \mapsto(e h \xi t g)\left(\xi t g^{-1}\right) g\left(e h g^{-1}\right)(\xi h g), \\
(\sigma, \rho) \quad: \quad \mathcal{X} \rightarrow \mathcal{X}, & \\
(\gamma, \rho) \quad: \quad \mathcal{C} \rightarrow \mathcal{C}, &
\end{array}
$$

and these assignments determine group homomorphisms from the Whitehead group to these five endomorphism groups. The accompanying diagram shows the relationship between the various groups and homomorphisms.

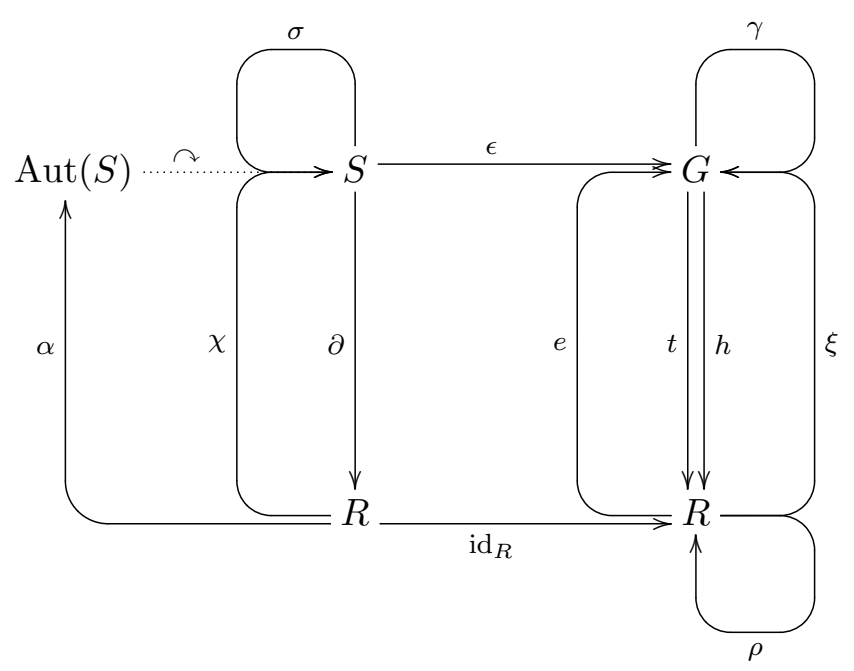


When axioms XMod 2: and Cat 2: are not satisfied, the corresponding structures are known as pre-crossed modules and pre-cat ${ }^{1}$-groups. In this case the Peiffer subgroup $P$ of $S$ is the subgroup of $\operatorname{ker}(\partial)$ generated by Peiffer commutators

$$
\llbracket s_{1}, s_{2} \rrbracket=\left(s_{1}^{-1}\right)^{\partial s_{2}} s_{2}^{-1} s_{1} s_{2} .
$$

Then $\mathcal{P}=\left(0: P \rightarrow\left\{1_{R}\right\}\right)$ is a normal sub-pre-crossed module of $\mathcal{X}$ and $\mathcal{X} / \mathcal{P}=(\partial: S / P \rightarrow R)$ is a crossed module. The restriction of $\epsilon: S \rightarrow R \ltimes S$ to $P$ is given by

$$
\epsilon \llbracket s_{1}, s_{2} \rrbracket=\left[\left(1_{R}, s_{1}^{\partial s_{2}}\right),\left(\left(\partial s_{2}\right)^{-1}, s_{2}\right)\right] \in[\operatorname{ker} t, \operatorname{ker} h] .
$$

The image $\epsilon P$ is the Peiffer subgroup [ker $t$, ker $h$ ] of $R \ltimes S$ and, if $\iota$ is the inclusion $\left\{1_{R}\right\} \rightarrow R$, then $\mathcal{C} /(\epsilon, \iota) \mathcal{P}=(e ; t, h:(R \ltimes S) / \epsilon P \rightarrow R)$ is the cat ${ }^{1}$-group corresponding to $\mathcal{X} / \mathcal{P}$. This construction is used when implementing induced cat ${ }^{1}$-groups.

The underlying groupoid $\mathcal{G}$ of a cat ${ }^{1}$-group $\mathcal{C}$ has the elements of $R$ as the set of objects and the elements of $G$ as arrows. The identity arrow at $r$ is $e r$. For each arrow $g$ the source(tail) is $t g$ and the target(head) is $h g$. Arrows $g, g^{\prime}$ are composable only when $h g=t g^{\prime}$, in which case the composite arrow is

$$
g * g^{\prime}=g\left(e t g^{-1}\right) g^{\prime}=g\left(e h g^{-1}\right) g^{\prime}
$$

with tail $t g$ and head $h g^{\prime}$. The groupoid inverse $\tilde{g}$ of $g$ for this composition is given by $\tilde{g}=$ $(e h g) g^{-1}(e t g)$ with $t \tilde{g}=h g, h \tilde{g}=t g, g * \tilde{g}=e t g$ and $\tilde{g} * g=e h g$. The equivalent formulae in $R \ltimes S$ are $(r, s) *\left(r(\partial s), s^{\prime}\right)=\left(r, s s^{\prime}\right)$ and $\widetilde{(r, s)}=\left(r(\partial s), s^{-1}\right)$.

Since $g^{-1}(e t g) \in \operatorname{ker} t$ and $(e h g) g^{-1} \in \operatorname{ker} h$, the map $g \mapsto \tilde{g}$ is an automorphism of $\mathcal{G}$ which restricts to the identity map on $e R$ and provides a cat ${ }^{1}$-isomorphism from $\mathcal{C}$ to the reverse cat ${ }^{1}$-group $\tilde{\mathcal{C}}=(e ; h, t: G \rightarrow R)$ of $\mathcal{C}$. The set of arrows out from $1_{R}$ is ker $t$ while the set of arrows in to $1_{R}$ is ker $h$, so ker $\partial$ is the set of loops at $1_{R}$. The set of objects in the component of $\mathcal{G}$ connected to $1_{R}$ is the image of $\partial$, so $\mathcal{G}$ is discrete when $\partial=0$.

\section{GAP implementation}

The group theory program GAP [20] is designed to facilitate the implementation of new structures as record types with their own output form. In version 3.4 of this package a separate operations record allows the overloading of functions such as kernel, centre and inner automorphism. We have developed a share package for GAP 3.4.4 containing some 160 functions for crossed modules, their morphisms and derivations; cat $^{1}$-groups, their morphisms and sections; and related constructions. All crossed modules and cat $^{1}$-groups require permutation groups as source and range, though groups of automorphisms, semidirect products and finitely presented groups are used by many of the functions. For each nonpermutation group we find it convenient to set up a pairing with an isomorphic permutation group. Thus, if $A$ is a group of automorphisms of a group $G$ and if $\theta$ is an isomorphism from $A$ to a permutation group $P$, an AutoPair for $A$ is a record pairA with fields pairA.auto $:=\mathbf{A}$, pair A.perm $:=\mathbf{P}$, pairA.a2p $:=\theta$, pairA.p2a $:=\theta^{-1}$ and pairA.isAutoPair $:=$ true. Such pairings are known in GAP4 as "nice isomorphisms". 
Also included are functions to compute the actor crossed module of a crossed module; and other crossed modules and morphisms in the actor square (see Norrie, $[18,19]$ ); and functions to calculate induced crossed modules (see Brown and Wensley, $[9,10,11])$ and induced cat ${ }^{1}$-groups ([1], Chapter $3)$.

A revised version of the package using GAP4 syntax is in preparation, in which the basic data structures are pre-crossed modules and pre-cat ${ }^{1}$-groups. Functions for constructing Peiffer subgroups; the underlying groupoid $\mathcal{G}$; crossed squares and their morphisms will be included.

We implement a crossed module $\mathcal{X}=(\partial: S \rightarrow R)$ as a record $\mathbf{X}$ with fields:

$\begin{array}{ll}\text { X.source, } & \text { the source group } S \text { of } \partial, \\ \text { X.boundary, } & \text { the homomorphsim } \partial, \\ \text { X.range, } & \text { the range group } R \text { of } \partial, \\ \text { X.aut, } & \text { a group of automorphisms of } S, \\ \text { X.action, } & \text { the homomorphism } \alpha \text { from } R \text { to X.aut, } \\ \text { X.isXMod, a boolean flag, normally true, } \\ \text { X.isDomain, always true, } \\ \begin{array}{ll}\text { X.operations, a special set of operations XModOps, } \\ \text { X.name, }\end{array} \quad \text { a concatenation of the names of } S \text { and } R \text {. }\end{array}$

Further fields, such as .isConjugationXMod, are added where appropriate.

The operations record XModOps includes functions for equality; size; list of elements; a special output form; and various functions for the actor square.

A morphism $(\sigma, \rho)$ of crossed modules is implemented as a record mor with fields:

$\begin{array}{ll}\text { mor.source, } & \text { the source crossed module } \mathbf{X}, \\ \text { mor.range, } & \text { the range crossed module } \mathbf{Y}, \\ \text { mor.sourceHom, } & \text { the homomorphism } \sigma \text { from } \mathbf{X} . \text { source to } \mathbf{Y} . \text { source, } \\ \text { mor.rangeHom, } & \text { the homomorphism } \rho \text { from } \mathbf{X . r a n g e} \text { to } \mathbf{Y} . r a n g e, \\ \text { mor.isXModMorphism, }, & \text { a Boolean flag, normally true, } \\ \text { mor.operations, } & \text { a special set of operations } \mathbf{X M o d M o r p h i s m O p s , ~} \\ \text { mor.name, } & \text { a concatenation of the names of } \mathbf{X} \text { and } \mathbf{Y} .\end{array}$

The operations record XModMorphismOps includes functions for equality; kernel and image; composite and inverse morphism; and tests such as IsEpimorphism.

A derivation $\chi: R \rightarrow S$ is defined in the same way that a group homomorphism is defined, by specifying a list of images for the generators of $R$ :

chi := XModDerivationByImages( R, S, R.generators, genimages ); .

If $w=r_{1} r_{2} \ldots r_{k}$ is an element of $R$ expressed as a word in the generators, then axiom Der 1: gives

$$
\chi w=\left(\chi r_{1}\right)^{r_{2} \ldots r_{k}}\left(\chi r_{2}\right)^{r_{3} \ldots r_{k}} \ldots\left(\chi r_{k-1}\right)^{r_{k}}\left(\chi r_{k}\right) .
$$

The function IsDerivation (see section 4.1) is used to test that such a $\chi$ is indeed a derivation. We store $\chi$ as a record chi with fields: source; range; generators; genimages; xmod; operations; isDerivation. 
We implement a cat $^{1}$-group $\mathcal{C}=(e ; t, h: G \rightarrow R)$ as a record $\mathbf{C}$ with fields: source; range; tail; head; embedRange; kernel; embedKernel; boundary; isDomain; operations; name; isCat1. A morphism mor $=(\gamma, \rho)$ of cat $^{1}$-groups is a record with fields similar to those of a morphism of crossed modules.

The functors providing the equvalence between the categories Cat1 and XMod are implemented as functions

- XModCat1(C), XModMorphismCat1Morphism(mor),

- Cat1XMod(X), Cat1MorphismXModMorphism(mor).

The second of these calculates the semidirect product $R \ltimes S$ and then finds a suitable isomorphic permutation group $G$ to act as the source, producing a SemidirectPair. In order to minimise the degree of $G$ it is preferable to start with $\mathcal{C}$, when a representation for $\mathcal{C}$ is known, and then construct $\mathcal{X}$.

There are two functions to determine the elements of the Whitehead group and the Whitehead monoid of $\mathcal{X}$, namely RegularDerivations and AllDerivations. If the whole monoid is needed at some stage, then the latter function should be used. A sub-record $\mathbf{D}=\mathbf{X}$.derivations of $\mathbf{X}$ is created which stores all the required information.

The functions WhiteheadMonoidTable and WhiteheadGroupTable calculate the multiplication tables of the monoid or group using the Whitehead multiplication, while WhiteheadPermGroup constructs a faithful, regular permutation representation of the group of regular derivations from the multiplication table.

The corresponding functions for sections are RegularSections and AllSections. Both create or modify a sub-record C.sections .

Example 3.1 Let $R$ be the symmetric group $S_{3}$ and $S$ its normal subgroup $C_{3}$. The conjugation crossed module $\mathcal{X}=\left(\iota: C_{3} \rightarrow S_{3}\right)$, the associated cat ${ }^{1}$-group, the derivation monoid of $\mathcal{X}$ and the actor crossed module are obtained as follows.

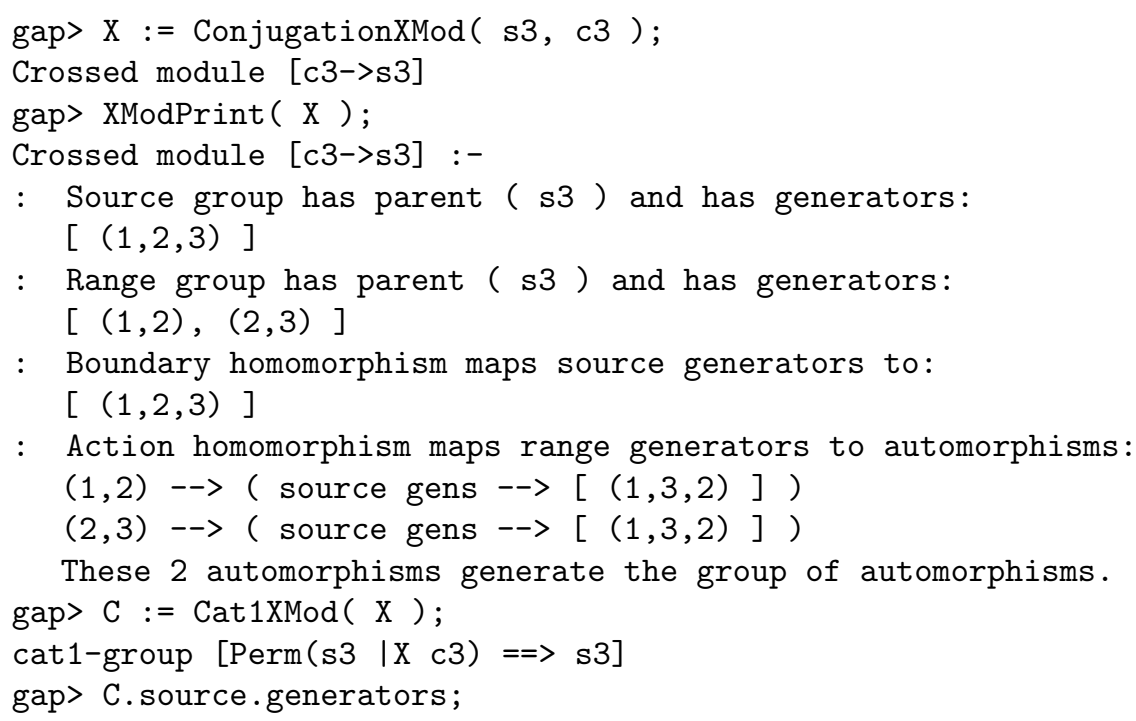


$[(2,3)(4,5),(2,3)(5,6),(1,2,3)]$

gap> D := AllDerivations ( $\mathrm{X}$ );

AllDerivations record for crossed module [c3->s3]

: 9 derivations found but unsorted.

gap> DerivationsSorted ( D );

true

gap> D.regular;

6

gap> PrintList ( WhiteheadMonoidTable( X ) );

$[1,2,3,4,5,6,7,8,9]$,

$[2,1,6,5,4,3,7,9,8]$,

$[3,4,1,2,6,5,8,7,9]$,

$[4,3,5,6,2,1,8,9,7]$,

$[5,6,4,3,1,2,9,8,7]$,

$[6,5,2,1,3,4,9,7,8]$,

$[7,7,7,7,7,7,7,7,7]$,

$[8,8,8,8,8,8,8,8,8]$,

$[9,9,9,9,9,9,9,9,9]]$

gap $>\mathrm{W}:=$ WhiteheadPermGroup $(\mathrm{X})$;

WG ( [c3->s3] )

gap> A := AutomorphismPermGroup ( X );

PermAut ( [c3->s3])

gap> Act := Actor $(\mathrm{X})$; ;

gap> XModPrint ( Act) ;

Crossed module Actor [c3->s3]

: Source group WG([c3->s3]) has generators:

$[(1,2)(3,4)(5,6),(1,3)(2,6)(4,5)]$

: Range group has parent ( PermAut(c3)xPermAut(s3)) and has generators:

$[(3,4,5),(1,2)(4,5)]$

: Boundary homomorphism maps source generators to:

$[(1,2)(3,5),(1,2)(4,5)]$

: Action homomorphism maps range generators to automorphisms:

$(3,4,5) \rightarrow$ ( source gens $\rightarrow[(1,5)(2,4)(3,6),(1,2)(3,4)(5,6)])$

$(1,2)(4,5)->$ ( source gens $->[(1,5)(2,4)(3,6),(1,3)(2,6)(4,5)])$

These 2 automorphisms generate the group of automorphisms.

\section{Outline algorithms}

In this section we comment on the algorithms used to construct derivations, sections, and the set of cat $^{1}$-structures on a group $G$.

\subsection{IsDerivation}

This function tests that a chosen set of images for the generators of $R$ does define a derivation. Let gen $R=\left\{r_{1}, r_{2}, \ldots, r_{m}\right\}$ be the generating set of $R$, and let $\left\{s_{1}, s_{2}, \ldots, s_{m}\right\}$ be the chosen images for $\chi$. First calculate the images $\chi\left(r_{i}^{-1}\right)=\left(\left(\chi r_{i}\right)^{r_{i}^{-1}}\right)^{-1}$. Construct an FpPair pair $R$ for $R$ with finitely presented group $F=$ pair $R$.fp having generating set $\left\{f_{1}, f_{2}, \ldots, f_{m}\right\}$ and pair $R . f 2 p$ mapping $f_{i}$ to $r_{i},(1 \leq i \leq m)$. For each relator rel in the presentation, with $w$ the corresponding word in genR, check that $\chi w=(\mathrm{l})$ using (4). When this is true, $\chi r$ is well-defined for all $r \in R$. Note that pair $R$ is stored as a field in the record $R$, so the pairing only has to be set up once. 


\subsection{RegularDerivations and AllDerivations}

The default method for calculating all the derivations of $\mathcal{X}$ is a simple backtrack algorithm. Let $\left\{R_{1}, R_{2}, \ldots, R_{m}=R\right\}$ be the sequence of subgroups of $R$ in which $R_{i}$ is generated by the first $i$ generators. The backtracking procedure constructs $m$-tuples of elements of $S$ as potential images for genR. As each $s_{i}=\chi r_{i}$ is chosen, with order $k_{i}$, the following are checked:

- $\rho_{i}: R_{i} \rightarrow R, \quad r_{j} \mapsto r_{j}\left(\partial s_{j}\right) \quad(1 \leq j \leq i)$ is a homomorphism,

- $s_{i}^{r_{i}^{k_{i}-1}} \ldots s_{i}^{r_{i}} s_{i}=\left(\right.$ ), which is the special case of (4) when $w=r_{i}^{k_{i}}$.

If either test fails, that part of the backtrack tree is discarded. The IsDerivation function is called when a full set of images has been found. If only the regular derivations are needed, the corresponding $\rho: R \rightarrow R$ is tested to see whether it is an automorphism. Note that all the derivations are found, even when not required.

\subsection{RegularSections and AllSections}

If X.derivations already exists when C.sections is required (and conversely) the sections are quickly obtained using $\xi r=(e r)(\epsilon \chi r)$.

When X.derivations does not already exist, a different method is used by default to calculate sections of $\mathcal{C}$. A section $\xi$ is determined by the choice of $s_{i}=\chi r_{i}$ for each $r_{i}$ in gen $R$. Since $r^{-1}(\rho r)=\partial \chi r$ it follows that $\chi r \in \partial^{-1}\left(r^{-1}(\rho r)\right)$. In order to find all regular sections, we use the standard GAP function AutomorphismGroup to obtain $\operatorname{Aut}(R)$. For each $\rho \in \operatorname{Aut}(R)$, lists of preimages

$$
\left[\partial^{-1}\left(r_{1}^{-1}\left(\rho r_{1}\right)\right), \partial^{-1}\left(r_{2}^{-1}\left(\rho r_{2}\right)\right), \ldots, \partial^{-1}\left(r_{m}^{-1}\left(\rho r_{m}\right)\right)\right]
$$

are constructed, and a backtrack procedure is used to select $s_{1}, s_{2}, \ldots, s_{m}$ from these lists, with each selection being tested to see whether it provides a partial homomorphism $R \rightarrow G$. Only the regular sections are found by this method.

A similar strategy is used to find all the sections, replacing $\operatorname{Aut}(R)$ by the endomorphism monoid $\operatorname{End}(R)$. Since no standard GAP function yet exists for computing $\operatorname{End}(R)$, we have added a function EndomorphismClasses.

\subsection{EndomorphismClasses}

An endomorphism $\varepsilon$ of $R$ is determined by

- a normal subgroup $N$ of $R$ and a faithful permutation representation of the quotient $\theta: R / N \rightarrow$ $Q$, giving a projection $\theta \circ \nu: R \rightarrow Q$ where $\nu: R \rightarrow R / N$ is the natural homomorphism;

- an automorphism $\alpha$ of $Q$;

- a subgroup $H^{\prime}$ in a conjugacy class $[H]$ of subgroups of $R$ isomorphic to $Q$ having representative $H$; an isomorphism $\phi: Q \cong H$; and a conjugating element $c \in R$ such that $H^{c}=H^{\prime}$. 
Then $\varepsilon$ takes values $\varepsilon r=(\phi \alpha \theta \nu r)^{c}$.

Endomorphisms are placed in the same class if they have the same choice of $N$ and $[H]$, so the number of endomorphisms is

$$
|\operatorname{End}(R)|=\sum_{\text {classes }}|\operatorname{Aut}(Q)||[H]|
$$

The function returns a record $\mathbf{E}=\mathbf{R}$.endomorphismClasses having a subfield .classes which is a list of records with fields:

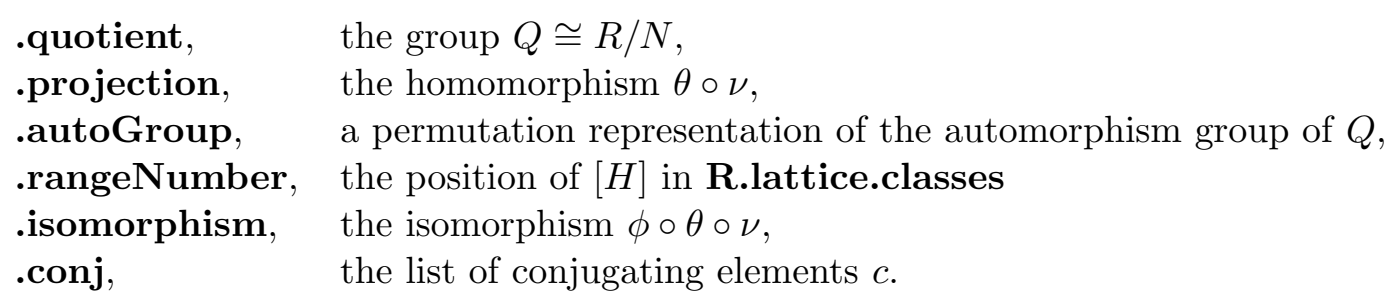

\subsection{AllCat1s}

A list $A$ of cat $^{1}$-groups with source $G$ is initialised, containing (id;id, id : $\left.G \rightarrow G\right)$ and $(1 ; 0 ; 0: G \rightarrow$ $\left.\left\{1_{G}\right\}\right)$. A list of representatives $\left\{H_{1}, H_{2}, \ldots, H_{m}\right\}$ of the non-trivial conjugacy classes of subgroups of $G$ is selected. (It would be more efficient to choose a set of representatives from the poset of automorphism classes of subgroups, rather than the conjugacy poset - particularly when $G$ is abelian.) For each $H_{i}$, all the idempotent endomorphisms $\phi: G \rightarrow H_{i}$ are constructed, and the images $\phi g_{k}$ of the generators $g_{k}$ of $G$ stored in a list $L_{i}$. These $\phi$ are candidates for the tail and head maps, so from each ordered pair of images in $L_{i}$ homomorphisms $t, h$ are constructed. If [ker $\left.t, \operatorname{ker} h\right]$ is trivial, then $t, h$ determine a cat $^{1}$-group $\mathcal{C}$. This $\mathcal{C}$ is compared with the entries in $A$ already obtained, using an AreIsomorphicCat1s function, and is added to $A$ if no isomorphism is found.

\subsection{Comparative timings}

We now present average times, in seconds, for the calculation of derivations and sections for six crossed modules, using the six standard constructions listed in section 2, each having $|G=R \ltimes S|=288$. Computations were performed on a DEC3000 Model 300LX Digital Alpha 64-bit workstation running xgap with $20 \mathrm{M}$ memory. Details of the six examples used are shown in the following table.

For each of the six crossed modules $\mathcal{X}$ three calls were made to each of the following functions.

- AllDerivations(X), creating a field X.derivations.

- X.derivations deleted, then RegularDerivations(X) executed. This takes a little longer since the irregular derivations are constructed and discarded.

- $\mathbf{C}:=\mathbf{C a t 1 X M o d}(\mathbf{X})$ called to construct the cat $^{1}$-group $\mathcal{C}$.

- X.derivations deleted, then RegularSections(C) executed. 
Table 1: Six examples of crossed modules

\begin{tabular}{|c|c|c|c|c|c|}
\hline No. & Type & Source & Range & im $\partial$ & degree of $S, R, G$ \\
\hline 1 & Conjugation & $\mathrm{a} 4$ & $\mathrm{~s} 4$ & $\mathrm{a} 4$ & $4,4,12$ \\
2 & Inner automorphism & $\mathrm{q} 24$ & $\mathrm{~d} 12$ & $\mathrm{~d} 12$ & $11,8,24$ \\
3 & RModule & $\mathrm{c} 6{ }^{2}$ & $\mathrm{~d} 8$ & $\mathrm{I}$ & $12,4,12$ \\
4 & Trivial action & $\mathrm{c} 12$ & $\mathrm{c} 8 \ltimes \mathrm{c} 3$ & $\mathrm{c} 4$ & $12,11,23$ \\
5 & Central extension & $\mathrm{sl}(2,3)$ & $\mathrm{a} 4$ & $\mathrm{a} 4$ & $11,4,24$ \\
6 & Direct product & $\mathrm{c} 3 \times \mathrm{c} 8$ & $\mathrm{~s} 3 \times \mathrm{c} 2$ & $\mathrm{c} 3$ & $11,5,29$ \\
\hline
\end{tabular}

- RegularDerivations(X) called again. This time, the existing list of sections is converted to a list of derivations.

- X.derivations, C.sections and C.range.automorphismGroup deleted, then AllSections(C) executed.

In order to eliminate one cause of fluctuating times, a garbage collection was performed before each call. Execution times were recorded, and the following averages noted.

Table 2: Execution times in seconds

\begin{tabular}{|c|c|rrrrrr|}
\hline \multirow{3}{*}{ No. } & {$[S \rightarrow R]$} & All & Reg. & Cat1 & Reg. & Reg. & All \\
\hline 1 & {$[a 4 \rightarrow s 4]$} & 40 & 44 & 34 & 8 & 0.2 & 18 \\
2 & {$[q 24 \rightarrow d 12]$} & 35 & 40 & 26 & 7.5 & 0.4 & 29 \\
3 & {$\left[c 6^{2} \rightarrow d 8\right]$} & 298 & 292 & 42 & 89 & 0.8 & 95 \\
4 & {$[c 12 \rightarrow c 8 \ltimes c 3]$} & 2.4 & 2.1 & 0.3 & 6.8 & 0.04 & 9.5 \\
5 & {$[s l(2,3) \rightarrow a 4]$} & 35 & 40 & 46 & 8 & 0.2 & 10 \\
6 & {$[c 3 \rightarrow s 3] \times[c 8 \rightarrow c 2]$} & 275 & 283 & 42 & 23 & 1.2 & 42 \\
\hline
\end{tabular}

Note that, given $\mathcal{C}$, the algorithm of 4.3 is quicker that that of 4.2 except in the fourth example. However in Examples 1,2,5, starting with $\mathcal{X}$, algorithm 4.2 finds all the derivations more quickly, due to the time required to construct a permutation representation of $G=R \ltimes S$.

\section{$5 \quad$ Table of cat $^{1}$-structures}

In the following table the 92 groups of size $\leq 30$ are ordered by their GAP number. For each group $G$ we list the size of $\operatorname{End}(G)$; the size of the set $\operatorname{IE}(G)$ of idempotents in $\operatorname{End}(G)$, which are candidates for $t$ and $h$; the size of $\mathcal{C}(G)$, the set of all cat ${ }^{1}$-structures on $G$; and the number of isomorphism classes of cat $^{1}$-structures. For each $G$ the first cat ${ }^{1}$-structure is $\mathcal{C}=(\mathrm{id}$; id, id : $G \rightarrow G$ ), corresponding to $\mathcal{X}=(0: I \rightarrow G)$, and we omit this from the list. These are the only cat $^{1}$-structures for the trivial 
group; the quaternion groups $q 8, q 16$; and the special linear group $s l(2,3)$. For each of the remaining isomorphism classes we list the names of $S, R$ and, when $t \neq h$ and $\partial \neq 0$, the kernel of the boundary. Just 52 of these 350 structures have $\partial \neq 0$. 
Table 3: Isomorphism classes of cat $^{1}$-structures

\begin{tabular}{|c|c|c|c|c|c|c|}
\hline GAP\# & $G$ & $|\operatorname{End}(G)|$ & $|\operatorname{IE}(G)|$ & $|\mathcal{C}(G)|$ & $|\mathcal{C}| \cong \mid$ & Names of $S, R$ and ker $\partial$ \\
\hline $1 / 1$ & I & 1 & 1 & 1 & 1 & \\
\hline $2 / 1$ & $\mathrm{c} 2$ & 2 & 2 & 2 & 2 & {$[\mathrm{c} 2, \mathrm{I}]$} \\
\hline $3 / 1$ & c3 & 3 & 2 & 2 & 2 & {$[\mathrm{c} 3, \mathrm{I}]$} \\
\hline $4 / 1$ & $\mathrm{k} 4$ & 16 & 8 & 14 & 4 & {$[\mathrm{k} 4, \mathrm{I}],[\mathrm{c} 2, \mathrm{c} 2],[\mathrm{c} 2, \mathrm{c} 2, \mathrm{I}]$} \\
\hline $4 / 2$ & $\mathrm{c} 4$ & 4 & 2 & 2 & 2 & {$[\mathrm{c} 4, \mathrm{I}]$} \\
\hline $5 / 1$ & $\mathrm{c} 5$ & 5 & 2 & 2 & 2 & {$[\mathrm{c} 5, \mathrm{I}]$} \\
\hline $6 / 1$ & c6 & 6 & 4 & 4 & 4 & {$[\mathrm{c} 6, \mathrm{I}],[\mathrm{c} 3, \mathrm{c} 2],[\mathrm{c} 2, \mathrm{c} 3]$} \\
\hline $6 / 2$ & s3 & 10 & 5 & 4 & 2 & {$[\mathrm{c} 3, \mathrm{c} 2]$} \\
\hline $7 / 1$ & $\mathrm{c} 7$ & 7 & 2 & 2 & 2 & {$[\mathrm{c} 7, \mathrm{I}]$} \\
\hline $8 / 1$ & $\mathrm{c} 2^{3}$ & 512 & 58 & 226 & 6 & $\begin{array}{l}{\left[\mathrm{c} 2^{3}, \mathrm{I}\right],[\mathrm{k} 4, \mathrm{c} 2],[\mathrm{k} 4, \mathrm{c} 2, \mathrm{c} 2]} \\
{[\mathrm{c} 2, \mathrm{k} 4],[\mathrm{c} 2, \mathrm{k} 4, \mathrm{I}]}\end{array}$ \\
\hline $8 / 2$ & $\mathrm{c} 4 \mathrm{c} 2$ & 32 & 10 & 18 & 6 & $\begin{array}{l}{[\mathrm{c} 4 \mathrm{c} 2, \mathrm{I}],[\mathrm{c} 4, \mathrm{c} 2],[\mathrm{c} 4, \mathrm{c} 2, \mathrm{c} 2],} \\
{[\mathrm{c} 2, \mathrm{c} 4],[\mathrm{c} 2, \mathrm{c} 4, \mathrm{I}]}\end{array}$ \\
\hline $8 / 3$ & $\mathrm{c} 8$ & 8 & 2 & 2 & 2 & {$[\mathrm{c} 8, \mathrm{I}]$} \\
\hline $8 / 4$ & $\mathrm{~d} 8$ & 36 & 10 & 9 & 3 & {$[\mathrm{c} 4, \mathrm{c} 2],[\mathrm{k} 4, \mathrm{c} 2]$} \\
\hline $8 / 5$ & q8 & 28 & 2 & 1 & 1 & \\
\hline $9 / 1$ & $c 3^{2}$ & 81 & 14 & 38 & 4 & {$\left[\mathrm{c} 3^{2}, \mathrm{I}\right],[\mathrm{c} 3, \mathrm{c} 3],[\mathrm{c} 3, \mathrm{c} 3, \mathrm{I}]$} \\
\hline $9 / 2$ & c9 & 9 & 2 & 2 & 2 & {$[\mathrm{c} 9, \mathrm{I}]$} \\
\hline $10 / 1$ & $\mathrm{c} 10$ & 10 & 4 & 4 & 4 & {$[\mathrm{c} 10, \mathrm{I}],[\mathrm{c} 5, \mathrm{c} 2],[\mathrm{c} 2, \mathrm{c} 5]$} \\
\hline $10 / 2$ & $\mathrm{~d} 10$ & 26 & 7 & 6 & 2 & {$[\mathrm{c} 5, \mathrm{c} 2]$} \\
\hline $11 / 1$ & $\mathrm{c} 11$ & 11 & 2 & 2 & 2 & {$[\mathrm{c} 11, \mathrm{I}]$} \\
\hline $12 / 1$ & $\mathrm{c} 6 \mathrm{c} 2$ & 48 & 16 & 28 & 8 & $\begin{array}{l}{[\mathrm{c} 6 \mathrm{c} 2, \mathrm{I}],[\mathrm{c} 6, \mathrm{c} 2],[\mathrm{c} 6, \mathrm{c} 2, \mathrm{c} 3],[\mathrm{k} 4, \mathrm{c} 3],} \\
{[\mathrm{c} 3, \mathrm{k} 4],[\mathrm{c} 2, \mathrm{c} 6],[\mathrm{c} 2, \mathrm{c} 6, \mathrm{I}]}\end{array}$ \\
\hline $12 / 2$ & c12 & 12 & 4 & 4 & 4 & {$[\mathrm{c} 12, \mathrm{I}],[\mathrm{c} 4, \mathrm{c} 3],[\mathrm{c} 3, \mathrm{c} 4]$} \\
\hline $12 / 3$ & $\mathrm{~d} 12$ & 64 & 21 & 12 & 4 & {$[\mathrm{c} 6, \mathrm{c} 2],[\mathrm{c} 3, \mathrm{k} 4],[\mathrm{c} 2, \mathrm{~s} 3]$} \\
\hline $12 / 4$ & q12 & 20 & 5 & 4 & 2 & {$[\mathrm{c} 3, \mathrm{c} 4]$} \\
\hline $12 / 5$ & a4 & 33 & 6 & 5 & 2 & {$[\mathrm{k} 4, \mathrm{c} 3]$} \\
\hline $13 / 1$ & $\mathrm{c} 13$ & 13 & 2 & 2 & 2 & {$[\mathrm{c} 13, \mathrm{I}]$} \\
\hline $14 / 1$ & c14 & 14 & 4 & 4 & 4 & {$[\mathrm{c} 14, \mathrm{I}],[\mathrm{c} 7, \mathrm{c} 2],[\mathrm{c} 2, \mathrm{c} 7]$} \\
\hline $14 / 2$ & $\mathrm{~d} 14$ & 50 & 9 & 8 & 2 & {$[\mathrm{c} 7, \mathrm{c} 2]$} \\
\hline $15 / 1$ & $\mathrm{c} 15$ & 15 & 4 & 4 & 4 & {$[\mathrm{c} 15, \mathrm{I}],[\mathrm{c} 5, \mathrm{c} 3],[\mathrm{c} 3, \mathrm{c} 5]$} \\
\hline $16 / 1$ & $c 2^{4}$ & 65536 & 382 & 4162 & 9 & $\begin{array}{l}{\left[\mathrm{c} 2^{4}, \mathrm{I}\right],\left[\mathrm{c} 2^{3}, \mathrm{c} 2\right],\left[\mathrm{c} 2^{3}, \mathrm{c} 2, \mathrm{k} 4\right],[\mathrm{k} 4, \mathrm{k} 4]} \\
{[\mathrm{k} 4, \mathrm{k} 4, \mathrm{c} 2],[\mathrm{k} 4, \mathrm{k} 4, \mathrm{I}],\left[\mathrm{c} 2, \mathrm{c} 2^{3}\right],\left[\mathrm{c} 2, \mathrm{c} 2^{3}, \mathrm{I}\right]}\end{array}$ \\
\hline $16 / 2$ & $\mathrm{c} 4 \mathrm{k} 4$ & 1024 & 82 & 322 & 12 & $\begin{array}{l}{[\mathrm{c} 4 \mathrm{k} 4, \mathrm{I}],[\mathrm{c} 4 \mathrm{c} 2, \mathrm{c} 2],[\mathrm{c} 4 \mathrm{c} 2, \mathrm{c} 2, \mathrm{c} 4],[\mathrm{c} 4 \mathrm{c} 2, \mathrm{c} 2, \mathrm{k} 4],} \\
{[\mathrm{c} 4, \mathrm{k} 4],[\mathrm{c} 4, \mathrm{k} 4, \mathrm{c} 2],[\mathrm{k} 4, \mathrm{c} 4],[\mathrm{k} 4, \mathrm{c} 4, \mathrm{c} 2],} \\
{[\mathrm{c} 2, \mathrm{c} 4 \mathrm{c} 2],[\mathrm{c} 2, \mathrm{c} 4 \mathrm{c} 2, \mathrm{I}],[\mathrm{c} 2, \mathrm{c} 4 \mathrm{c} 2, \mathrm{I}]}\end{array}$ \\
\hline $16 / 3$ & $\mathrm{c} 8 \mathrm{c} 2$ & 64 & 10 & 18 & 6 & $\begin{array}{l}{[\mathrm{c} 8 \mathrm{c} 2, \mathrm{I}],[\mathrm{c} 8, \mathrm{c} 2],[\mathrm{c} 8, \mathrm{c} 2, \mathrm{c} 4]} \\
{[\mathrm{c} 2, \mathrm{c} 8],[\mathrm{c} 2, \mathrm{c} 8, \mathrm{I}]}\end{array}$ \\
\hline $16 / 4$ & $c 4^{2}$ & 256 & 26 & 98 & 5 & {$\left[\mathrm{c} 4^{2}, \mathrm{I}\right],[\mathrm{c} 4, \mathrm{c} 4],[\mathrm{c} 4, \mathrm{c} 4, \mathrm{c} 2],[\mathrm{c} 4, \mathrm{c} 4, \mathrm{I}]$} \\
\hline $16 / 5$ & $\mathrm{c} 16$ & 16 & 2 & 2 & 2 & {$[\mathrm{c} 16, \mathrm{I}]$} \\
\hline $16 / 6$ & $\mathrm{~d} 8 \mathrm{c} 2$ & 1088 & 82 & 97 & 9 & $\begin{array}{l}{[\mathrm{c} 4 \mathrm{c} 2, \mathrm{c} 2],\left[\mathrm{c} 2^{3}, \mathrm{c} 2\right],[\mathrm{c} 4, \mathrm{k} 4],[\mathrm{c} 4, \mathrm{k} 4, \mathrm{c} 2]} \\
{[\mathrm{k} 4, \mathrm{k} 4],[\mathrm{k} 4, \mathrm{k} 4, \mathrm{c} 2],[\mathrm{c} 2, \mathrm{~d} 8],[\mathrm{c} 2, \mathrm{~d} 8, \mathrm{I}]}\end{array}$ \\
\hline $16 / 7$ & q8c2 & 448 & 18 & 17 & 3 & {$[\mathrm{c} 2, \mathrm{q} 8],[\mathrm{c} 2, \mathrm{q} 8, \mathrm{I}]$} \\
\hline $16 / 8$ & $\mathrm{~d} 8 \mathrm{y} 4$ & 224 & 26 & 13 & 2 & {$[\mathrm{c} 4 \mathrm{c} 2, \mathrm{c} 2]$} \\
\hline $16 / 9$ & $\mathrm{c} 2 \ltimes \mathrm{c} 4 \mathrm{c} 2$ & 128 & 18 & 25 & 4 & {$[\mathrm{c} 4 \mathrm{c} 2, \mathrm{c} 2],[\mathrm{k} 4, \mathrm{c} 4],[\mathrm{k} 4, \mathrm{c} 4, \mathrm{c} 2]$} \\
\hline $16 / 10$ & $\mathrm{c} 4 \ltimes \mathrm{c} 4$ & 96 & 10 & 17 & 3 & {$[\mathrm{c} 4, \mathrm{c} 4],[\mathrm{c} 4, \mathrm{c} 4, \mathrm{c} 2]$} \\
\hline $16 / 11$ & $\mathrm{c} 2 \ltimes \mathrm{c} 8$ & 48 & 6 & 5 & 2 & {$[\mathrm{c} 8, \mathrm{c} 2]$} \\
\hline $16 / 12$ & $\mathrm{~d} 16$ & 100 & 18 & 9 & 2 & {$[\mathrm{c} 8, \mathrm{c} 2]$} \\
\hline $16 / 13$ & qd16 & 52 & 10 & 5 & 2 & {$[\mathrm{c} 8, \mathrm{c} 2]$} \\
\hline $16 / 14$ & q16 & 36 & 2 & 1 & 1 & \\
\hline $17 / 1$ & c17 & 17 & 2 & 2 & 2 & {$[\mathrm{c} 17, \mathrm{I}]$} \\
\hline $18 / 1$ & c6c3 & 162 & 28 & 76 & 8 & $\begin{array}{l}{[\mathrm{c} 6 \mathrm{c} 3, \mathrm{I}],\left[\mathrm{c} 3^{2}, \mathrm{c} 2\right],[\mathrm{c} 6, \mathrm{c} 3],[\mathrm{c} 6, \mathrm{c} 3, \mathrm{c} 2]} \\
{[\mathrm{c} 3, \mathrm{c} 6],[\mathrm{c} 3, \mathrm{c} 6, \mathrm{I}],\left[\mathrm{c} 2, \mathrm{c} 3^{2}\right]}\end{array}$ \\
\hline $18 / 2$ & $\mathrm{c} 18$ & 18 & 4 & 4 & 4 & {$[\mathrm{c} 18, \mathrm{I}],[\mathrm{c} 9, \mathrm{c} 2],[\mathrm{c} 2, \mathrm{c} 9]$} \\
\hline $18 / 3$ & d18 & 82 & 11 & 10 & 2 & {$[\mathrm{c} 9, \mathrm{c} 2]$} \\
\hline
\end{tabular}




\begin{tabular}{|c|c|c|c|c|c|c|}
\hline GAP\# & $G$ & $|\operatorname{End}(G)|$ & $|\operatorname{IE}(G)|$ & $|\mathcal{C}(G)|$ & $|\mathcal{C}| \cong 1$ & Names of $S, R$ and ker $\partial$ \\
\hline $18 / 4$ & s3c3 & 36 & 12 & 8 & 4 & {$\left[\mathrm{c}^{2}, \mathrm{c} 2\right],[\mathrm{c} 3, \mathrm{c} 6],[\mathrm{c} 3, \mathrm{~s} 3]$} \\
\hline $18 / 5$ & $\mathrm{c} 2 \ltimes \mathrm{c} 3^{2}$ & 730 & 47 & 118 & 4 & {$\left[\mathrm{c} 3^{2}, \mathrm{c} 2\right],[\mathrm{c} 3, \mathrm{~s} 3],[\mathrm{c} 3, \mathrm{~s} 3, \mathrm{I}]$} \\
\hline $19 / 1$ & c19 & 19 & 2 & 2 & 2 & {$[\mathrm{c} 19, \mathrm{I}]$} \\
\hline $20 / 1$ & c10c2 & 80 & 16 & 28 & 8 & $\begin{array}{l}{[\mathrm{c} 10 \mathrm{c} 2, \mathrm{I}],[\mathrm{c} 10, \mathrm{c} 2],[\mathrm{c} 10, \mathrm{c} 2, \mathrm{c} 5],[\mathrm{c} 5, \mathrm{k} 4],} \\
{[\mathrm{k} 4, \mathrm{c} 5],[\mathrm{c} 2, \mathrm{c} 10],[\mathrm{c} 2, \mathrm{c} 10, \mathrm{I}]}\end{array}$ \\
\hline $20 / 2$ & c20 & 20 & 4 & 4 & 4 & {$[\mathrm{c} 20, \mathrm{I}],[\mathrm{c} 5, \mathrm{c} 4],[\mathrm{c} 4, \mathrm{c} 5]$} \\
\hline $20 / 3$ & $\mathrm{~d} 20$ & 144 & 31 & 18 & 4 & {$[\mathrm{c} 10, \mathrm{c} 2],[\mathrm{c} 5, \mathrm{k} 4],[\mathrm{c} 2, \mathrm{~d} 10]$} \\
\hline $20 / 4$ & q20 & 52 & 7 & 6 & 2 & {$[\mathrm{c} 5, \mathrm{c} 4]^{]}$} \\
\hline $20 / 5$ & $\mathrm{c} 4 \ltimes \mathrm{c} 5$ & 36 & 7 & 6 & 2 & {$[\mathrm{c} 5, \mathrm{c} 4]$} \\
\hline $21 / 1$ & $\mathrm{c} 21$ & 21 & 4 & 4 & 4 & {$[\mathrm{c} 21, \mathrm{I}],[\mathrm{c} 7, \mathrm{c} 3],[\mathrm{c} 3, \mathrm{c} 7]$} \\
\hline $21 / 2$ & $\mathrm{c} 3 \ltimes \mathrm{c} 7$ & 57 & 9 & 8 & 2 & {$[\mathrm{c} 7, \mathrm{c} 3]$} \\
\hline $22 / 1$ & $\mathrm{c} 22$ & 22 & 4 & 4 & 4 & {$[\mathrm{c} 22, \mathrm{I}],[\mathrm{c} 11, \mathrm{c} 2],[\mathrm{c} 2, \mathrm{c} 11]$} \\
\hline $22 / 2$ & $\mathrm{~d} 22$ & 122 & 13 & 12 & 2 & {$[\mathrm{c} 11, \mathrm{c} 2]$} \\
\hline $23 / 1$ & c23 & 23 & 2 & 2 & 2 & {$[\mathrm{c} 23, \mathrm{I}]$} \\
\hline $24 / 1$ & $\mathrm{c} 6 \mathrm{k} 4$ & 1536 & 116 & 452 & 12 & $\begin{array}{l}{[\mathrm{c} 6 \mathrm{k} 4, \mathrm{I}],[\mathrm{c} 6 \mathrm{c} 2, \mathrm{c} 2],[\mathrm{c} 6 \mathrm{c} 2, \mathrm{c} 2, \mathrm{c} 6],\left[\mathrm{c} 2{ }^{3}, \mathrm{c} 3\right]} \\
{[\mathrm{c} 6, \mathrm{k} 4],[\mathrm{c} 6, \mathrm{k} 4, \mathrm{c} 3],[\mathrm{k} 4, \mathrm{c} 6],[\mathrm{k} 4, \mathrm{c} 6, \mathrm{c} 2]} \\
{\left[\mathrm{c} 3, \mathrm{c} 22^{3}\right],[\mathrm{c} 2, \mathrm{c} 6 \mathrm{c} 2],[\mathrm{c} 2, \mathrm{c} 6 \mathrm{c} 2, \mathrm{I}]}\end{array}$ \\
\hline $24 / 2$ & $c 12 c 2$ & 96 & 20 & 36 & 12 & $\begin{array}{l}{[\mathrm{c} 12 \mathrm{c} 2, \mathrm{I}],[\mathrm{c} 12, \mathrm{c} 2],[\mathrm{c} 12, \mathrm{c} 2, \mathrm{c} 6],[\mathrm{c} 4 \mathrm{c} 2, \mathrm{c} 3]} \\
{[\mathrm{c} 6, \mathrm{c} 4],[\mathrm{c} 6, \mathrm{c} 4, \mathrm{c} 3],[\mathrm{c} 4, \mathrm{c} 6],[\mathrm{c} 4, \mathrm{c} 6, \mathrm{c} 2]} \\
{[\mathrm{c} 3, \mathrm{c} 4 \mathrm{c} 2],[\mathrm{c} 2, \mathrm{c} 12],[\mathrm{c} 2, \mathrm{c} 12, \mathrm{I}]}\end{array}$ \\
\hline $24 / 3$ & $\mathrm{c} 24$ & 4 & 4 & 4 & 4 & {$[\mathrm{c} 24, \mathrm{I}],[\mathrm{c} 8, \mathrm{c} 3],[\mathrm{c} 3, \mathrm{c} 8]$} \\
\hline $24 / 4$ & $\mathrm{~d} 8 \mathrm{c} 3$ & 108 & 20 & 18 & 6 & {$[\mathrm{c} 12, \mathrm{c} 2],[\mathrm{c} 6 \mathrm{c} 2, \mathrm{c} 2],[\mathrm{c} 4, \mathrm{c} 6],[\mathrm{k} 4, \mathrm{c} 6],[\mathrm{c} 3, \mathrm{~d} 8]$} \\
\hline $24 / 5$ & q8c3 & 84 & 4 & 2 & 2 & {$[\mathrm{c} 3, \mathrm{q} 8]^{]}$} \\
\hline $24 / 6$ & s3k4 & 1792 & 157 & 116 & 8 & $\begin{array}{l}{[\mathrm{c} 6 \mathrm{c} 2, \mathrm{c} 2],[\mathrm{c} 6, \mathrm{k} 4],[\mathrm{c} 6, \mathrm{k} 4, \mathrm{c} 3],[\mathrm{k} 4, \mathrm{~s} 3]} \\
{\left[\mathrm{c} 3, \mathrm{c} 2^{3}\right],[\mathrm{c} 2, \mathrm{~d} 12],[\mathrm{c} 2, \mathrm{~d} 12, \mathrm{I}]}\end{array}$ \\
\hline $24 / 7$ & s3c4 & 128 & 27 & 12 & 4 & {$[\mathrm{c} 12, \mathrm{c} 2],[\mathrm{c} 4, \mathrm{~s} 3],[\mathrm{c} 3, \mathrm{c} 4 \mathrm{c} 2]$} \\
\hline $24 / 8$ & q12c2 & 160 & 25 & 36 & 6 & $\begin{array}{l}{[\mathrm{c} 6, \mathrm{c} 4],[\mathrm{c} 6, \mathrm{c} 4, \mathrm{c} 3],[\mathrm{c} 3, \mathrm{c} 4 \mathrm{c} 2]} \\
{[\mathrm{c} 2, \mathrm{q} 12],[\mathrm{c} 2, \mathrm{q} 12, \mathrm{I}]}\end{array}$ \\
\hline $24 / 9$ & $\mathrm{c} 8 \ltimes \mathrm{c} 3$ & 40 & 5 & 4 & 2 & {$[\mathrm{c} 3, \mathrm{c} 8]$} \\
\hline $24 / 10$ & $\mathrm{a} 4 \mathrm{c} 2$ & 72 & 15 & 10 & 4 & {$\left[\mathrm{c} 2^{3}, \mathrm{c} 3\right],[\mathrm{k} 4, \mathrm{c} 6],[\mathrm{c} 2, \mathrm{a} 4]$} \\
\hline $24 / 11$ & $\mathrm{~d} 8 \ltimes \mathrm{c} 3$ & 124 & 23 & 12 & 4 & {$[\mathrm{c} 6 \mathrm{c} 2, \mathrm{c} 2],[\mathrm{k} 4, \mathrm{~s} 3],[\mathrm{c} 3, \mathrm{~d} 8]$} \\
\hline $24 / 12$ & $\mathrm{~d} 24$ & 196 & 33 & 20 & 4 & {$[\mathrm{c} 12, \mathrm{c} 2],[\mathrm{c} 4, \mathrm{~s} 3],[\mathrm{c} 3, \mathrm{~d} 8]$} \\
\hline $24 / 13$ & q24 & 124 & 5 & 4 & 2 & {$[\mathrm{c} 3, \mathrm{q} 8]^{\top}$} \\
\hline $24 / 14$ & $\operatorname{sl}(2,3)$ & 33 & 6 & 1 & 1 & \\
\hline $24 / 15$ & s4 & 58 & 12 & 5 & 2 & {$[\mathrm{k} 4, \mathrm{~s} 3]$} \\
\hline $25 / 1$ & $c 5^{2}$ & 625 & 32 & 152 & 4 & {$\left[\mathrm{c} 5^{2}, \mathrm{I}\right],[\mathrm{c} 5, \mathrm{c} 5],[\mathrm{c} 5, \mathrm{c} 5, \mathrm{I}]$} \\
\hline $25 / 2$ & c25 & 25 & 2 & 2 & 2 & {$[\mathrm{c} 25, \mathrm{I}]$} \\
\hline $26 / 1$ & $\mathrm{c} 26$ & 26 & 4 & 4 & 4 & {$[\mathrm{c} 26, \mathrm{I}],[\mathrm{c} 13, \mathrm{c} 2],[\mathrm{c} 2, \mathrm{c} 13]$} \\
\hline $26 / 2$ & $\mathrm{~d} 26$ & 170 & 15 & 14 & 2 & {$[\mathrm{c} 13, \mathrm{c} 2]^{\mathrm{t}}$} \\
\hline $27 / 1$ & $c 3^{3}$ & 19683 & 236 & 2108 & 6 & $\begin{array}{l}{\left[\mathrm{c}^{3}, \mathrm{I}\right],\left[\mathrm{c} 3^{2}, \mathrm{c} 3\right],\left[\mathrm{c} 3^{2}, \mathrm{c} 3, \mathrm{c} 3\right]} \\
{\left[\mathrm{c} 3, \mathrm{c} 3^{2}\right],\left[\mathrm{c} 3, \mathrm{c} 3^{2}, \mathrm{I}\right]}\end{array}$ \\
\hline $27 / 2$ & c9c3 & 243 & 20 & 56 & 6 & $\begin{array}{l}{[\mathrm{c} 9 \mathrm{c} 3, \mathrm{I}],[\mathrm{c} 9, \mathrm{c} 3],[\mathrm{c} 9, \mathrm{c} 3, \mathrm{c} 3],} \\
{[\mathrm{c} 3, \mathrm{c} 9],[\mathrm{c} 3, \mathrm{c} 9, \mathrm{I}]}\end{array}$ \\
\hline $27 / 3$ & $\mathrm{c} 27$ & 27 & 2 & 2 & 2 & {$[\mathrm{c} 27, \mathrm{I}]$} \\
\hline $27 / 4$ & $\mathrm{c} 3 \ltimes \mathrm{c} 3^{2}$ & 729 & 38 & 37 & 2 & {$\left[\mathrm{c} 3^{2}, \mathrm{c} 3\right]$} \\
\hline $27 / 5$ & $\mathrm{c} 3 \ltimes \mathrm{c} 9$ & 135 & 11 & 10 & 2 & {$[\mathrm{c} 9, \mathrm{c} 3]$} \\
\hline $28 / 1$ & c14c2 & 112 & 16 & 28 & 8 & $\begin{array}{l}{[\mathrm{c} 14 \mathrm{c} 2, \mathrm{I}],[\mathrm{c} 14, \mathrm{c} 2],[\mathrm{c} 14, \mathrm{c} 2, \mathrm{c} 7],[\mathrm{c} 7, \mathrm{k} 4],} \\
{[\mathrm{k} 4, \mathrm{c} 7],[\mathrm{c} 2, \mathrm{c} 14],[\mathrm{c} 2, \mathrm{c} 14, \mathrm{I}]}\end{array}$ \\
\hline $28 / 2$ & $\mathrm{c} 28$ & 28 & 4 & 4 & 4 & {$[\mathrm{c} 28, \mathrm{I}],[\mathrm{c} 7, \mathrm{c} 4],[\mathrm{c} 4, \mathrm{c} 7]$} \\
\hline $28 / 3$ & $\mathrm{~d} 28$ & 256 & 41 & 24 & 4 & {$[\mathrm{c} 14, \mathrm{c} 2],[\mathrm{c} 7, \mathrm{k} 4],[\mathrm{c} 2, \mathrm{~d} 14]$} \\
\hline $28 / 4$ & q28 & 100 & 9 & 8 & 2 & {$[\mathrm{c} 7, \mathrm{c} 4]$} \\
\hline $29 / 1$ & $\mathrm{c} 29$ & 29 & 2 & 2 & 2 & {$[\mathrm{c} 29, \mathrm{I}]$} \\
\hline $30 / 1$ & c30 & 30 & 8 & 8 & 8 & $\begin{array}{l}{[\mathrm{c} 30, \mathrm{I}],[\mathrm{c} 15, \mathrm{c} 2],[\mathrm{c} 10, \mathrm{c} 3],[\mathrm{c} 6, \mathrm{c} 5],} \\
{[\mathrm{c} 5, \mathrm{c} 6],[\mathrm{c} 3, \mathrm{c} 10],[\mathrm{c} 2, \mathrm{c} 15]}\end{array}$ \\
\hline $30 / 2$ & $\mathrm{~d} 10 \mathrm{c} 3$ & 78 & 14 & 12 & 4 & {$[\mathrm{c} 15, \mathrm{c} 2],[\mathrm{c} 5, \mathrm{c} 6],[\mathrm{c} 3, \mathrm{~d} 10]$} \\
\hline $30 / 3$ & $\mathrm{~d} 6 \mathrm{c} 5$ & 50 & 10 & 8 & 4 & {$[\mathrm{c} 15, \mathrm{c} 2],[\mathrm{c} 5, \mathrm{~s} 3],[\mathrm{c} 3, \mathrm{c} 10]$} \\
\hline $30 / 4$ & $\mathrm{~d} 30$ & 226 & 25 & 24 & 4 & {$[\mathrm{c} 15, \mathrm{c} 2],[\mathrm{c} 5, \mathrm{~s} 3],[\mathrm{c} 3, \mathrm{~d} 10]$} \\
\hline
\end{tabular}


Example 5.1 The data in the table is stored as a list Cat1List, so any cat ${ }^{1}$-group in the table may be selected using the Cat1Select function. In the first call only the group $G$ is specified, while in the second call a third parameter is supplied.

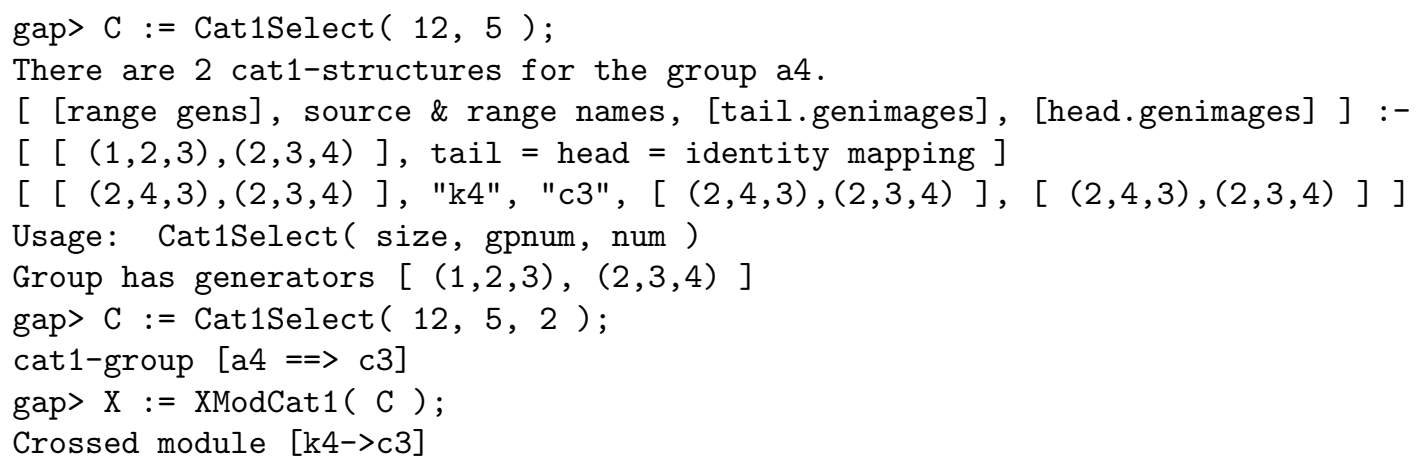

\section{References}

[1] M Alp, GAP, crossed modules, cat ${ }^{1}$-groups: Applications of computational group theory, Ph.D. Thesis, University of Wales, Bangor, 1997.

[2] M Alp and C D Wensley, XMOD - Crossed modules and Cat1-groups in GAP, version 1.3 Manual for the XMOD share package (1997), 1-78.

[3] Z Arvasi and T Porter, Simplicial and crossed resolutions of commutative algebras, J. Algebra 181 (1996), 426-448.

[4] R Brown, Higher dimensional group theory in Low-dimensional topology, London Math. Soc. Lecture Note Series 48, ed. R Brown and T L Thickstun, Cambridge University Press, 1982, pp.215-238.

[5] R Brown, From groups to groupoids: a brief survey, Bull. London Math. Soc. 19 (1987), 113-134.

[6] R Brown and P J Higgins, On the connection between the second relative homotopy group and some related spaces, Proc. London. Math. Soc. 36 (1978), 193-212.

[7] R Brown and J-L Loday, Van Kampen theorems for diagram of spaces, Topology 26 (1987), 311-335.

[8] R Brown and C Spencer, $\mathcal{G}$-groupoids, crossed modules and the fundamental groupoid of a topological group, Nede. Akad. Wetensch. Proc. 79 (1976), 296-302.

[9] R Brown and C D Wensley, On finite induced crossed modules, and the homotopy 2-type of mapping cones, Theory and Applications of Categories 1 (1995), 54-71.

[10] R Brown and C D Wensley, Computing crossed modules induced by an inclusion of a normal subgroup, with applications to homotopy 2-types, Theory and Applications of Categories 2 (1996), $3-16$. 
[11] R Brown and C D Wensley, On the computation of induced crossed modules, U.W.Bangor Preprint 97.04 (1997).

[12] W H Cockroft, On two-dimensional aspherical complexes, Proc. London Math. Soc. (3) 4 (1954), 375-384.

[13] G J Ellis, Crossed modules and their higher dimensional analogues, Ph.D. Thesis, University of Wales, Bangor, 1984.

[14] G J Ellis and R Steiner, Higher dimensional crossed modules and the homotopy groups of $(n+1)$ ads. J. Pure and Appl. Algebra 46 (1987), 117-136.

[15] N D Gilbert, Derivations, Automorphisms and crossed modules, Comm. in Algebra 18 (1990), 2703-2734.

[16] J-L Loday, Spaces with finitely many non-trivial homotopy groups, J. App. Algebra 24 (1982), 179-202.

[17] A S-T Lue, Semi-complete crossed modules and holomorphs of groups, Bull. London Math. Soc. 11 (1979), 8-16.

[18] K J Norrie, Actions and automorphisms of crossed modules, Bull. Soc. Math. France 118 (1990), 129-146.

[19] K J Norrie, Crossed module and anologues of group theorems, Ph.D. Thesis, King's College, University of London, 1987.

[20] M Schönert et al, GAP-Groups, Algorithms, and Programming, Lehrstuhl D für Mathematik, Rheinisch Westfälische Technische Hochschule, Aachen, Germany, fifth edition, 1995.

[21] J H C Whitehead, Combinatorial homotopy II, Bull. Amer. Math. Soc. 55 (1949), 453-496.

[22] J H C Whitehead, On operators in relative homotopy groups, Ann. of Math. 49 (1948), 610-640. 